\title{
Sequential Interval Estimation of a Location Parameter with the Fixed Width in the Non-regular Case
}

\author{
Dedicated to Professor Masafumi Akahira on his 60th birthday \\ Ken-ichi Koike \\ Institute of Mathematics, University of Tsukuba \\ Tsukuba, Ibaraki, Japan
}

\begin{abstract}
For a location-scale parameter family of distributions with a finite support, a sequential confidence interval with a fixed width is obtained for the location parameter, and its asymptotic consistency and efficiency are shown. Some comparisons with the Chow-Robbins procedure are also done.
\end{abstract}

Keywords: Coverage probability; Extreme value; Non-regular case; Sequential interval estimation.

Subject Classifications: 62L12;62F25.

\section{INTRODUCTION}

Suppose that we are to estimate a location parameter $\theta$ of a sequence of random observations $X_{1}, X_{2}, \ldots, X_{n}, \ldots$ with unknown scale $\xi$. We would like to obtain sequentially a confidence interval of fixed width $2 d$ with confidence coefficient $1-\alpha$. Obviously we can not obtain a fixed sample size procedure if $\xi$ is unknown. There are many works on the fixed-width interval estimation of normal mean (see, e.g. Ghosh et al. (1997)).

Address correspondence to Ken-ichi Koike, Institute of Mathematics, University of Tsukuba, 1-1-1 Tennodai, Tsukuba, Ibaraki 305-8571, Japan; Fax: 81-29-853-6501; E-mail: koike@math.tsukuba.ac.jp 
Suppose that $X_{1}, X_{2}, \ldots, X_{n}, \ldots$ is a sequence of independent and identically distributed (i.i.d.) random variables according to the uniform distribution on the interval $(\theta-(\xi / 2), \theta+(\xi / 2))$, where $\theta\left(\in \mathbb{R}^{1}\right)$ and $\xi(>0)$ are unknown. Let $X_{(1)}:=\min _{1 \leq i \leq n} X_{i}, X_{(n)}:=\max _{1 \leq i \leq n} X_{i}$. Then the midrange and the range are $M_{n}:=\left(X_{(1)}+X_{(n)}\right) / 2, R_{n}:=X_{(n)}-X_{(1)}$, respectively. Akahira and Koike (2005) considered a stopping rule:

$$
\tau_{1}:=\inf \left\{\begin{array}{l|l}
n \geq n_{0} & \frac{R_{n}}{n-1} \leq-\frac{2 d}{\log \alpha}
\end{array}\right\}
$$

where $n_{0}(\geq 2)$ is an initial size of sample. They showed the asymptotic consistency and efficiency of the estimation procedure $\left(\tau_{1},\left[M_{\tau_{1}}-d, M_{\tau_{1}}+d\right]\right)$.

In this paper, we consider the case of a location-scale parameter family of distributions with a finite support on the interval $(\theta-\xi a, \theta+\xi a)$, where $\theta$ and $\xi$ are unknown, and obtain a sequential confidence interval of $\theta$ with fixed width $2 d$ and confidence coefficient $1-\alpha$, and show its asymptotic consistency and efficiency. Some comparisons with the Chow-Robbins procedure are also done.

\section{ASYMPTOTIC DISTRIBUTIONS OF THE EXTREME VAL- UES}

In this section we consider the asymptotic distributions of the extreme values for distributions with a finite support, in a similar way to Akahira (1991) and Akahira and Takeuchi (1995).

Let $Z_{1}, Z_{2}, \ldots$, be a sequence of independent and identically distributed (i.i.d.) random variables according to the density function $f_{0}(x-\theta)\left(\theta \in \mathbb{R}^{1}\right)$ with respect to the Lebesgue measure. We assume the following conditions: (A1) $f_{0}(x)$ has a finite support $(-a, a)^{1}(a>0)$, i.e., $f_{0}(x)>0$ for $-a<x<a$, and $f_{0}(x)=0$ otherwise.

(A2) $f_{0}(x)$ is continuously differentiable in the open interval $(-a, a)$ and

$$
\lim _{x \rightarrow-a+0} f_{0}(x)=c, \lim _{x \rightarrow a-0} f_{0}(x)=c^{\prime},
$$

\footnotetext{
${ }^{1}$ If the support of $f_{0}$ is $(-a, b)(a \neq b)$, then the normalized midrange does not converge to $\theta$ in probability as $n \rightarrow \infty$.
} 
where $c$ and $c^{\prime}$ are some positive constants.

(A3) $f_{0}(x)$ satisfies

$$
\begin{aligned}
& f_{0}(x) \approx g(x+a)^{\gamma} \quad(x \rightarrow-a+0), \\
& f_{0}(x) \approx g^{\prime}|x-a|^{\gamma} \quad(x \rightarrow a-0),
\end{aligned}
$$

where $\gamma, g$ and $g^{\prime}$ are some positive constants ${ }^{2}$.

Putting $Z_{(1)}:=\min _{1 \leq i \leq n} Z_{i}, Z_{(n)}:=\max _{1 \leq i \leq n} Z_{i}, U:=n\left(Z_{(1)}+a-\theta\right)$ and $V:=n\left(Z_{(n)}-a-\theta\right)$, we have the following lemma (cf. Akahira (1991), Akahira and Takeuchi (1995)).

Lemma 1. Under the conditions (A1) and (A2), the joint(j.) p.d.f. $f_{U, V}^{(n)}(u, v)$ of $(U, V)$ satisfies

$$
f_{U, V}^{(n)}(u, v) \rightarrow \begin{cases}c c^{\prime} \exp \left\{c^{\prime} v-c u\right\} & (v<0<u), \\ 0 & (\text { otherwise }) .\end{cases}
$$

as $n \rightarrow \infty$.

Proof. The j.p.d.f. $f_{U, V}^{(n)}(u, v)$ of $(U, V)$ is

$$
\begin{aligned}
& f_{U, V}^{(n)}(u, v) \\
= & \left\{\begin{array}{rr}
\frac{n-1}{n}\left\{F\left(a+\frac{v}{n}\right)-F\left(-a+\frac{u}{n}\right)\right\}^{n-2} f_{0}\left(-a+\frac{u}{n}\right) f_{0}\left(a+\frac{v}{n}\right) \\
(v<0<u),
\end{array}\right. \\
0 & \text { (otherwise), }
\end{aligned}
$$

where $F(x)=\int_{-\infty}^{x} f_{0}(u) d u$. Hence, by its expansion, we have the desired result.

Next, we consider the location-scale parameter family of distributions with a finite support $(\theta-\xi a, \theta+\xi a)$. Suppose that $X_{1}, X_{2}, \ldots, X_{n}, \ldots$ is a sequence of i.i.d. random variables with the p.d.f. $(1 / \xi) f_{0}((x-\theta) / \xi)$, where $\theta \in \mathbb{R}$ and $\xi>0$. Put $Y_{i}:=\left(X_{i}-\theta\right) / \xi$ for each $i=1,2, \ldots$, and $Y_{(1)}:=$ $\min _{1 \leq i \leq n} Y_{i}, Y_{(n)}:=\max _{1 \leq i \leq n} Y_{i}$. Letting $S:=n\left(Y_{(1)}+Y_{(n)}\right) / 2$ and $T=$ $n\left(Y_{(1)}-Y_{(n)}+2 a\right) / 2$, we have the asymptotic (as.) j.p.d.f. of $(S, T)$

$$
f_{S, T}(s, t)= \begin{cases}2 c c^{\prime} \exp \left\{-\left(c-c^{\prime}\right) s-\left(c+c^{\prime}\right) t\right\} & (t>|s|), \\ 0 & \text { (otherwise) } .\end{cases}
$$

\footnotetext{
${ }^{2}$ If the converging order $\gamma$ is different, then the normalized midrange does not converge to $\theta$ in probability as $n \rightarrow \infty$.
} 
Then the as. marginal(m.) p.d.f.'s of $S$ and $T$ are given by

$$
\begin{aligned}
f_{S}(s) & = \begin{cases}K e^{-2 c s} & (s \geq 0), \\
K e^{2 c^{\prime} s} & (s<0),\end{cases} \\
f_{T}(t) & = \begin{cases}\frac{2 c c^{\prime}}{c^{\prime}-c}\left(e^{-2 c t}-e^{-2 c^{\prime} t}\right) & \left(t>0 \text { and } c \neq c^{\prime}\right), \\
4 c^{2} t e^{-2 c t} & \left(t>0 \text { and } c=c^{\prime}\right), \\
0 & (\text { otherwise })\end{cases}
\end{aligned}
$$

respectively, where $K=2 c c^{\prime} /\left(c+c^{\prime}\right)$.

In the case when $\lim _{x \rightarrow-a+0} f_{0}(x)=\lim _{x \rightarrow a-0} f_{0}(x)=0$, we need another lemma. Putting $U^{\prime}:=n^{1 /(\gamma+1)}\left(Z_{(1)}+a-\theta\right)$ and $V^{\prime}:=n^{1 /(\gamma+1)}\left(Z_{(n)}-a-\theta\right)$, we have the following lemma in a similar way to Lemma 1.

Lemma 2. Under the conditions (A1) and (A3), the j.p.d.f.

$f_{U^{\prime}, V^{\prime}}^{(n)}(u, v)$ of $\left(U^{\prime}, V^{\prime}\right)$ satisfies

$$
f_{U^{\prime}, V^{\prime}}^{(n)}(u, v) \rightarrow \begin{cases}g g^{\prime}(-u v)^{\gamma} \exp \left\{-\frac{g^{\prime}}{\gamma+1}(-v)^{\gamma+1}-\frac{g}{\gamma+1} u^{\gamma+1}\right\} & (v<0<u), \\ 0 & (\text { otherwise }) .\end{cases}
$$

as $n \rightarrow \infty$.

The proof is omitted since it is similar to the one of Lemma 1.

From Lemma 2, $U^{\prime}$ and $\left(-V^{\prime}\right)$ are asymptotically, independently distributed according to Weibull distributions.

\section{CONSTRUCTING CONFIDENCE INTERVAL}

In this section we construct a sequential confidence interval for $\theta$. In the first place, we consider the case under the conditions (A1) and (A2). For $0<\alpha<1$, let $l_{0}$ be the solution ${ }^{3}$ of $l$ for the equation

$$
\frac{c+c^{\prime}}{c c^{\prime}} \alpha=\frac{e^{-2 c l}}{c}+\frac{e^{-2 c^{\prime} l}}{c^{\prime}} .
$$

\footnotetext{
${ }^{3}$ It can be shown easily that such $l_{0}$ exists uniquely.
} 
If $\xi$ is known, we have from (2.2) that

$$
\begin{aligned}
P\left\{\left|M_{n}-\theta\right| \leq d\right\} & =P\left\{n\left|M_{n}-\theta\right| / \xi \leq d n / \xi\right\} \\
& \approx \int_{-d n / \xi}^{d n / \xi} f_{S}(s) d s \\
& =1-\frac{c c^{\prime}}{c+c^{\prime}}\left(\frac{e^{-2 c n d / \xi}}{c}+\frac{e^{-2 c^{\prime} n d / \xi}}{c^{\prime}}\right),
\end{aligned}
$$

where " $\approx$ " means that the distribution of $n\left|M_{n}-\theta\right| / \xi$ is approximated by the asymptotic distribution. Letting $n^{*}=l_{0} \xi / d$, we have for $n \geq n^{*}$

$$
1-\frac{c c^{\prime}}{c+c^{\prime}}\left(\frac{e^{-2 c n d / \xi}}{c}+\frac{e^{-2 c^{\prime} n d / \xi}}{c^{\prime}}\right) \geq 1-\alpha .
$$

$n^{*}$ is referred as the asymptotically optimal size of samples if $\xi$ is known. Note that $n\left(M_{n}-\theta\right) / \xi=S$ and $R_{n} / \xi=-(T / n)+2 a$. Now we take as the stopping rule

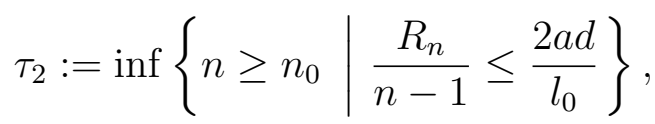

where $n_{0}(\geq 2)$ is an initial size of sample. Then we obtain the asymptotic properties of the estimation procedure $\left(\tau_{2},\left[M_{\tau_{2}}-d, M_{\tau_{2}}+d\right]\right)$ as follows.

Theorem 1. For the sequential estimation procedure $\left(\tau_{2},\left[M_{\tau_{2}}-d, M_{\tau_{2}}+d\right]\right)$, the following hold.

(i) $\lim _{d \rightarrow 0+} P\left\{\left|M_{\tau_{2}}-\theta\right| \leq d\right)=1-\alpha \quad$ (asymptotic consistency).

(ii) $\tau_{2} / n^{*} \stackrel{\text { a.s. }}{\rightarrow} 1 \quad(d \rightarrow 0+)$.

(iii) $E\left(\tau_{2}\right) / n^{*} \rightarrow 1(d \rightarrow 0+) \quad$ (asymptotic efficiency).

Proof. (i) From Lemma 1 of Chow and Robbins (1965), the stopping rule $\tau_{2}$ given by (3.1) satisfies

$$
\lim _{d \rightarrow 0+} \frac{d \tau_{2}}{\xi l_{0}}=1 \quad \text { a.s. }
$$

Since $S=n\left(M_{n}-\theta\right) / \xi$ converges in distribution to a distribution with the density given by (2.2) as $n \rightarrow \infty$, it follows from Theorem 1 of Anscombe (1952) that $\tau_{2}\left(M_{\tau_{2}}-\theta\right)$ converges in distribution to the same distribution as $d \rightarrow 0+$. Hence, since $d \tau_{2} / \xi \stackrel{\text { a.s. }}{\rightarrow} l_{0}$ as $d \rightarrow 0+$ from (3.2), it follows that

$$
\begin{aligned}
\lim _{d \rightarrow 0+} P\left\{\left|M_{\tau_{2}}-\theta\right| \leq d\right\} & =\lim _{d \rightarrow 0+} P\left\{\tau_{2}\left|M_{\tau_{2}}-\theta\right| / \xi \leq d \tau_{2} / \xi\right\} \\
& =\int_{-l_{0}}^{l_{0}} f_{S}(s) d s=1-\alpha .
\end{aligned}
$$


(ii) From (3.2) and the definition of $l_{0}$, we have $\tau_{2} / n^{*}=\tau_{2} d /\left(l_{0} \xi\right) \stackrel{\text { a.s. }}{\rightarrow} 1$ as $d \rightarrow 0+$.

(iii) From Lemma 2 of Chow and Robbins (1965), we have the desired result.

Remark. In particular, if $c=c^{\prime}$, then $l_{0}=-\log \alpha /(2 c)$ and $\tau_{2}$ given in (3.1) is expressed as

$$
\tau_{2}=\inf \left\{\begin{array}{l|l}
n \geq n_{0} & \frac{R_{n}}{n-1} \leq-\frac{4 a c d}{\log \alpha}
\end{array}\right\}
$$

which is equal to $\tau_{1}$ when the underlying distribution is uniform distribution on the interval $(\theta-(\xi / 2), \theta+(\xi / 2))$.

In the second place, we compare this with the Chow-Robbins procedure. Let $X_{1}, X_{2}, \ldots$ be a sequence of i.i.d. random variables with the mean $\theta$ and the variance $\sigma^{2}$. Let $\bar{X}_{n}:=\sum_{i=1}^{n} X_{i} / n, s_{n}^{2}=\sum_{i=1}^{n}\left(X_{i}-\bar{X}_{n}\right)^{2} /(n-1)$. Chow and Robbins (1965) considered a stopping rule defined by

$$
\tau_{C R}:=\inf \left\{n \geq n_{0} \mid n \geq u_{\alpha / 2}^{2} d^{-2} s_{n}^{2}\right\},
$$

where $u_{\alpha / 2}$ is the upper $\alpha / 2$ point of $N(0,1)$ and $n_{0}(\geq 2)$ is an initial size of samples. They showed the asymptotic consistency and efficiency of the estimation procedure $\left(\tau_{C R},\left[\bar{X}_{\tau_{C R}}-d, \bar{X}_{\tau_{C R}}+d\right]\right)$.

Since, from Theorem 2.2 of Akahira and Koike (2005), Theorem 1 and Theorem of Chow and Robbins (1965),

$$
\tau_{1} \approx \frac{\log \alpha}{\log (1-(2 d / \xi))} \approx \frac{-\xi \log \alpha}{2 d}, \quad \tau_{2} \approx l_{0} \xi / d, \quad \tau_{C R} \approx u_{\alpha / 2}^{2} \sigma^{2} / d^{2},
$$

as $d \rightarrow 0+$, we have $\tau_{1} / \tau_{C R}, \tau_{2} / \tau_{C R} \rightarrow 0(d \rightarrow 0+)$. Therefore $\tau_{1}, \tau_{2}$ is asymptotically better than $\tau_{C R}$ in the sense of the average size of sample.

Furthermore, we consider the case under the conditions (A1) and (A3). By putting $S^{\prime}:=n^{1 /(\gamma+1)}\left(Y_{(1)}+Y_{(n)}\right) / 2$ and $T^{\prime}:=n^{1 /(\gamma+1)}\left(Y_{(1)}-Y_{(n)}+2 a\right) / 2$, the as.j.p.d.f. of $\left(S^{\prime}, T^{\prime}\right)$ and the as.m.p.d.f.'s of $S^{\prime}$ and $T^{\prime}$ are obtained from Lemma 2 . In a similar way to (3.3), we take $l_{0}$ satisfying $\int_{-l_{0}}^{l_{0}} f_{S^{\prime}}(s) d s=1-\alpha$ for the as.m.p.d.f. $f_{S^{\prime}}(s)$ of $S^{\prime}$. 
If $\xi$ is known, we have

$$
\begin{aligned}
P\left\{\left|M_{n}-\theta\right| \leq d\right\} & =P\left\{n^{1 /(\gamma+1)}\left|M_{n}-\theta\right| / \xi \leq d n^{1 /(\gamma+1)} / \xi\right\} \\
& \approx \int_{-d n^{1 /(\gamma+1)} / \xi}^{d n^{1 /(\gamma+1)} / \xi} f_{S^{\prime}}(s) d s,
\end{aligned}
$$

where " $\approx$ " means that the distribution of $n^{1 /(\gamma+1)}\left|M_{n}-\theta\right| / \xi$ is approximated by the asymptotic distribution. The optimal size of sample required for attaining the preassigned coverage probability $1-\alpha$ is the smallest positive integer $\geq\left(l_{0} \xi / d\right)^{\gamma+1}=: n^{* *}$ (say). Define a stopping rule as

$$
\tau_{3}:=\inf \left\{\begin{array}{l|l}
n \geq n_{0} & \frac{R_{n}}{n^{1 /(\gamma+1)}} \leq \frac{2 a d}{l_{0}}
\end{array}\right\},
$$

where $n_{0}(\geq 2)$ is an initial size of samples. Then the next theorem follows.

Theorem 2. For the sequential estimation procedure $\left(\tau_{3},\left[M_{\tau_{3}}-d, M_{\tau_{3}}+d\right]\right)$, the following hold.

(i) $\lim _{d \rightarrow 0+} P\left\{\left|M_{\tau_{3}}-\theta\right| \leq d\right)=1-\alpha \quad$ (asymptotic consistency).

(ii) $\tau_{3} / n^{* *} \stackrel{\text { a.s. }}{\rightarrow} 1 \quad(d \rightarrow 0+)$.

(iii) $E\left(\tau_{3}\right) / n^{* *} \rightarrow 1(d \rightarrow 0+) \quad$ (asymptotic efficiency).

Proof. The proof for (i) is similar to the one of Theorem 1 (i). (ii) follows from $\left(\tau_{3} / n^{* *}\right)^{1 /(\gamma+1)} \stackrel{\text { a.s. }}{\rightarrow} 1$ as $d \rightarrow 0+$.

(iii) From (ii), by Fatou's lemma,

$$
\liminf _{d \rightarrow 0+} \frac{E\left(\tau_{3}\right)}{n^{* *}} \geq E\left(\liminf _{d \rightarrow 0+} \frac{\tau_{3}}{n^{* *}}\right)=1
$$

On the other hand, since $0 \leq R_{n} \leq 2 a \xi$ with probability 1 for any $n \in \mathbb{N}$, we have $0 \leq\left(R_{n} l_{0} /(2 a d)\right)^{\gamma+1} \leq\left(2 a \xi l_{0} /(2 a d)\right)^{\gamma+1}=\left(l_{0} \xi / d\right)^{\gamma+1}$ with probability 1 for any $n \in \mathbb{N}$. So, $0 \leq\left(R_{n} l_{0} /(2 a d)\right)^{\gamma+1} \leq n$ with probability 1 for $n$ satisfying $n \geq\left(l_{0} \xi / d\right)^{\gamma+1}+1$. Therefore, since $\tau_{3}=$ inf $\left\{n \geq n_{0} \mid\left(R_{n} l_{0} /(2 a d)\right)^{\gamma+1} \leq n\right\}$, we have $\tau_{3} \leq\left(\frac{l_{0} \xi}{d}\right)^{\gamma+1}+1$. Then, using the definition of $n^{* *}$, we have

$$
\frac{E\left(\tau_{3}\right)}{n^{* *}} \leq\left\{\left(\frac{l_{0} \xi}{d}\right)^{\gamma+1}+1\right\}\left(\frac{l_{0} \xi}{d}\right)^{-(\gamma+1)}=1+\left(\frac{d}{l_{0} \xi}\right)^{\gamma+1}
$$


hence

$$
\limsup _{d \rightarrow 0+} \frac{E\left(\tau_{3}\right)}{n^{* *}} \leq 1
$$

Combining (3.4) and (3.5), we obtain (iii).

From Theorem 2 and Theorem of Chow and Robbins (1965), $\tau_{3} \approx$ $\left(l_{0} \xi / d\right)^{\gamma+1}$ and $\tau_{C R} \approx u_{\alpha / 2}^{2} \sigma^{2} / d^{2}$ as $d \rightarrow 0+$. Therefore,

$$
\tau_{3} / \tau_{C R} \begin{cases}=o(1) & (0<\gamma<1) \\ =O(1) & (\gamma=1) \\ \rightarrow \infty & (\gamma>1)\end{cases}
$$

as $d \rightarrow 0+$. Therefore, $\tau_{3}$ is asymptotically better than $\tau_{C R}$ in the sense of the average size of sample if $0<\gamma<1$.

In this paper, we considered the cases when the values at the endpoints of the support of the p.d.f. are positive simultaneously, or tend to 0 at the same speed. In the meantime, if the either value at the endpoints of the support of the p.d.f. is positive, or tend to 0 at a different speed, then the coefficients of $n^{\gamma}\left(X_{(1)}-a-\theta\right)$ and $n^{\delta}\left(X_{(n)}-b-\theta\right)$ converging to nontrivial random variables are different and estimation by using the midrange $M_{n}$ is inappropriate.

\section{NUMERICAL EXAMPLE}

In this section we examine the coverage probability of the procedure $\left[M_{\tau_{2}}-\right.$ $\left.d, M_{\tau_{2}}+d\right]$ by simulation based on 100000 repetitions. Suppose that

$X_{1}, X_{2}, \ldots, X_{n}, \ldots$ is a sequence of i.i.d. random variables with the p.d.f. $(1 / \xi) f_{0}((x-\theta) / \xi)$, where $\theta \in \mathbb{R}, \xi>0$ and $f_{0}(\cdot)$ is a trapezoid-shape p.d.f. given by

$$
f_{0}(x)= \begin{cases}\left(\frac{1}{2}-c\right) x+\frac{1}{2} & (x \in(-1,1)), \\ 0 & \text { (otherwise) }\end{cases}
$$

with $0<c<1$. Note that, $f_{0}$ is the p.d.f. of the uniform distribution over $(-1,1)$ and an asymmetric p.d.f. over $(-1,1)$ for $c=0.5$ and a sufficiently small $c>0$, respectively. Since $M_{\tau_{2}}$ is location equivariant, we may assume $\theta=0$ without loss of generality.

When $\alpha=0.10, d=0.01(0.01) 0.05, \xi=1(1) 5$ and $n_{0}=5$, Tables 1 and 2 show the values of coverage probabilities of the sequential estimation 
procedure $\left(\tau_{2},\left[M_{\tau_{2}}-d, M_{\tau_{2}}+d\right]\right)$ for $c=0.1$ and $c=0.5$, respectively. The result suggests that the estimation procedure is consistent for this case.

Table 1. Coverage probabilities of $\left[M_{\tau_{2}}-d, M_{\tau_{2}}+d\right]$ for $c=0.1$

\begin{tabular}{|c|c|c|c|c|c|}
\hline$\xi \backslash d$ & 0.01 & 0.02 & 0.03 & 0.04 & 0.05 \\
\hline 1 & 0.90637 & 0.91545 & 0.92348 & 0.93092 & 0.93758 \\
\hline 2 & 0.89830 & 0.90544 & 0.90960 & 0.91424 & 0.92017 \\
\hline 3 & 0.90123 & 0.90313 & 0.90713 & 0.90832 & 0.91030 \\
\hline 4 & 0.89926 & 0.90117 & 0.90333 & 0.90615 & 0.90804 \\
\hline 5 & 0.89817 & 0.89952 & 0.90318 & 0.90421 & 0.90561 \\
\hline
\end{tabular}

Table 2. Coverage probabilities of $\left[M_{\tau_{2}}-d, M_{\tau_{2}}+d\right]$ for $c=0.5$

\begin{tabular}{|c|c|c|c|c|c|}
\hline$\xi \backslash d$ & 0.01 & 0.02 & 0.03 & 0.04 & 0.05 \\
\hline 1 & 0.90210 & 0.90727 & 0.91183 & 0.91328 & 0.91988 \\
\hline 2 & 0.89929 & 0.90131 & 0.90330 & 0.90628 & 0.91176 \\
\hline 3 & 0.89849 & 0.89947 & 0.90221 & 0.90235 & 0.90525 \\
\hline 4 & 0.89729 & 0.89729 & 0.89982 & 0.90169 & 0.90322 \\
\hline 5 & 0.89785 & 0.8998 & 0.89906 & 0.89862 & 0.90054 \\
\hline
\end{tabular}

\section{ACKNOWLEDGMENTS}

The author would like to thank Professor M. Akahira of University of Tsukuba for his valuable comments throughout the preparation of the paper. The author would also like to thank anonymous referees for their helpful suggestions. This research was partially supported by the Ministry of Education, Science, Sports and Culture of Japan, Grant-in-Aid for Young Scientists (B) 14740062.

\section{REFERENCES}

Akahira, M. (1991). The 3/2th and 2nd Order Asymptotic Efficiency of Maximum Probability Estimators in Non-regular Cases, Annals of Institute of Statistical Mathematics 43: 181-195. 
Akahira, M. and Koike, K. (2005). Sequential Interval Estimation of a Location Parameter with the Fixed Width in the Uniform Distribution with an Unknown Scale Parameter, Sequential Analysis 24: 63-75.

Akahira, M. and Takeuchi, K. (1995). Non-Regular Statistical Estimation, Lecture Notes in Statistics 10\%, New York: Springer-Verlag.

Anscombe, F. J. (1952). Large Sample Theory of Sequential Estimation, Proceedings of Cambridge Philosophical Society 48: 600-607.

Chow, Y. S. and Robbins, H. (1965). On the Asymptotic Theory of Fixedwidth Sequential Confidence Intervals for the Mean, Annals of Mathematical Statistics 36: 457-462.

Ghosh, M., Mukhopadhyay, N., and Sen, P. K. (1997). Sequential Estimation, New York: Wiley.

Koike, K. and Akahira, M. (2004). Sequential Interval Estimation of a Location Parameter with the Fixed Width in a Class of Non-regular Distributions with an Unknown Scale Parameter (in Japanese), in Proceedings of Symposiums, Research Institute for Mathematical Sciences 1380, M. Akahira, ed., pp. 191-199, Kyoto: Kyoto University. 